\title{
Advances in the use of the Short-Time Fourier Transform for assessing urban trees' root systems
}

\author{
Livia Lantini*a, Fabio Tosti $^{\text {a }}$, Lilong Zou ${ }^{\text {a }}$, Luca Bianchini Ciampoli ${ }^{\text {b }}$, Amir M. Alani ${ }^{\mathrm{a}}$ \\ aSchool of Computing and Engineering, University of West London (UWL), St Mary's Road,

 \\ 62, 00146 Roma, Italy
}

\begin{abstract}
Urbanisation often leads to the destruction of green areas in the urban environment. To this extent, urban trees help mitigating its detrimental effects, as well as offering a variety of socioeconomic and environmental benefits. The presence of root systems in built environments usually results in structural damage, as for example shrinkage of expansive soils due to water suction by roots, resulting in subsidence and fissures in foundations, or roots obstructing pipes and sewers and damaging roads and pavements.

Ground Penetrating Radar (GPR) has been extensively used in various areas of civil and environmental engineering. Research has focused on implementing 3D algorithms and investigating root density, and a recent experimental research examined the feasibility of a novel tree root assessing methodology, that processes GPR data both in time and frequency domains. The aim of this research is to improve upon the above-mentioned data processing algorithm, investigating the variation of the frequency spectrum of the GPR signal in urban tree root systems' surveys by means of a Short-Time Fourier Transform (STFT). Results proved the viability of the methodology and paved the way to further developments for the investigation of urban trees' root systems using GPR.
\end{abstract}

Keywords: Ground Penetrating Radar (GPR), short-time Fourier transform (STFT), tree root mapping, urban trees

\section{INTRODUCTION}

More than half of the world's population lives in cities in this historical moment, and this figure is predicted to grow up to nearly 70 percent by the year $2050{ }^{1}$. The exponential growth of cities often lacks land-use planning policies, resulting in degradation of green areas in and around cities. If properly maintained, urban trees help mitigating the negative impact and social effects of urbanisation, thus making cities more resilient to these changes by offering a wide variety of socioeconomic and environmental benefits ${ }^{2-4}$. To this end, the UK government has recently announced the planting of 50,000 trees in England 5 .

However, the conflicting coexistence of tree root systems with the built environment often causes extensive structural damage with obvious socioeconomic implications. An example is the shrinkage of expansive soils due to water extraction by roots, leading to subsidence and cracks in building foundations ${ }^{6}$. Furthermore, pipes and sewers with localised water leakage can be obstructed by uncontrolled roots development, as these proliferate in areas with a high concentration of moisture levels ${ }^{7}$. Finally, tree roots can create cracking or uplifting of road pavements and pedestrian sidewalks, resulting in severe danger to vulnerable users such as pedestrians, cyclists and bikers ${ }^{8}$.

In this context, Ground Penetrating Radar (GPR) has long been proven to be an effective non-destructive testing (NDT) method in various areas of civil and environmental engineering ${ }^{9-12}$. With regards to forestry applications, GPR is becoming increasingly popular due to its efficiency and the possibility of retrieving accurate information on the subsoil, moisture level and the root systems in a completely non-intrusive way ${ }^{13-15}$. Recent research has focused on the implementation of automated algorithms for root mapping in a $3 \mathrm{D}$ environment ${ }^{16,17}$ and the investigation of root mass density ${ }^{18}$. In addition, GPR was proven effective for the assessment and mapping of tree root systems' architecture underneath road pavements ${ }^{19}$. Finally, a recent experimental study investigated the feasibility of a novel tree root assessment methodology, based on the processing of GPR data in time and frequency domains ${ }^{20}$.

The aim of this research is therefore to improve upon the latest developments in data processing algorithms based on combined time-frequency information, investigating the variation in the frequency spectrum vs propagation time response of the GPR signal for urban tree root systems. To this extent, the Short-Time Fourier Transform (STFT) is applied to the 
GPR data, following a dedicated pre-processing stage aimed at eliminating noise-related information and disturbances from manmade features (i.e., pavement layers). The strength of this methodology lies in its applicability to complex urban scenarios and special survey requirements, such as the need for expeditious surveys of street trees, which are often difficult to access. Furthermore, this method can find application in case more comprehensive assessment methods, such as the use of acquisition methods based on circular scans ${ }^{17}$, are not applicable.

\section{METHODOLOGY}

\subsection{Test Site and Equipment}

The survey was carried out as part of a research campaign within the premises of the University of West London, Ealing, London (United Kingdom) (Figure 1). A number of 17 linear scans, $50 \mathrm{~m}$ long and spaced $0.10 \mathrm{~m}$ from one another, were performed on the pavement next to a green area containing a row of trees. For the purposes of this research, the scan located closer to the green space was selected.

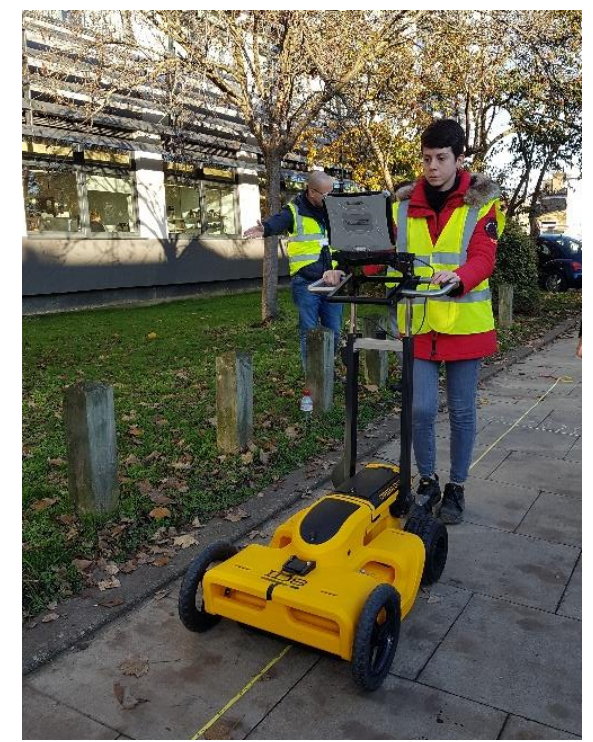

Figure 1. The GPR survey taking place at the University of West London, London, UK

The Opera Duo ground-coupled GPR system, manufactured by IDS GeoRadar (Part of Hexagon) and equipped with 700 $\mathrm{MHz}$ and $250 \mathrm{MHz}$ central frequency antennas, was used for surveying purposes. Data were collected using a time window of $80 \mathrm{~ns}$, discretised across 512 samples. The horizontal resolution was set to $3.06 \times 10^{-2} \mathrm{~m}$. To achieve the optimal effective resolution for the scope of this research, the analysis was limited to data collected using the $700 \mathrm{MHz}$ antenna only. This choice was due to the need to achieve a depth of investigation such as to analyse the whole tree root system, without excessively affecting the signal resolution.

\subsection{Data Processing}

The aim of this stage is the reduction of noise from the GPR data, as well as producing quantifiable information and easily interpretable images for a subsequent interpretation of results. To this end, data were processed following a multi-stage procedure, including filters working in both time and frequency domains as follows:

- Zero-offset removal

- Time-zero correction

- $\quad$ Time-varying gain

- Singular value decomposition (SVD) filter

- $\quad$ Band-pass filtering 
Subsequently, data were processed using a STFT function ${ }^{20}$. This method allows the frequency of local sections of a signal to be analysed as it changes over time, as follows:

$$
\operatorname{STFT}(t, \omega)=\int_{t}[x(t) \cdot w(\tau-t)] \cdot e^{-j \omega t} d t
$$

where STFT is the frequency energy at time $t$ and frequency $\omega, x$ is the reflected amplitude and $w$ is the window function. In practice, a signal is divided into shorter segments of equal length and then the Fourier transform is calculated separately for each segment.

\section{RESULTS AND DISCUSSION}

\subsection{Target Identification and Application Windows}

Figure 2 shows the selected B-scan after the application of the multi-stage signal processing procedure as described above. An area for the application of the STFT function was then selected (identified by the red square in Figure 2), as it includes a semi-horizontal layer crossing two reflection hyperbolas. This is likely to represent two adjacent roots growing at the intersection between pavement layers, a common situation in urban environments that can lead to pavement uplifting and compromise safety of drivers and pedestrians ${ }^{21,22}$.



Figure 2. The B-scan analysed in this study. The red square identifies the selected application area.

Figure 3 shows the selected area, which was then divided into 5 application windows. Such windows were designed to be comparable in size and as wide as the investigated hyperbolas. In more detail, each application window includes $11 \mathrm{~A}$ scans, covering about $0.34 \mathrm{~m}$ in length, for a total investigated longitudinal distance of $1.70 \mathrm{~m}$. The hyperbolas are contained in windows 2 and 4, respectively. Each window was finally reduced to its average signal value, for the subsequent application of the STFT function.
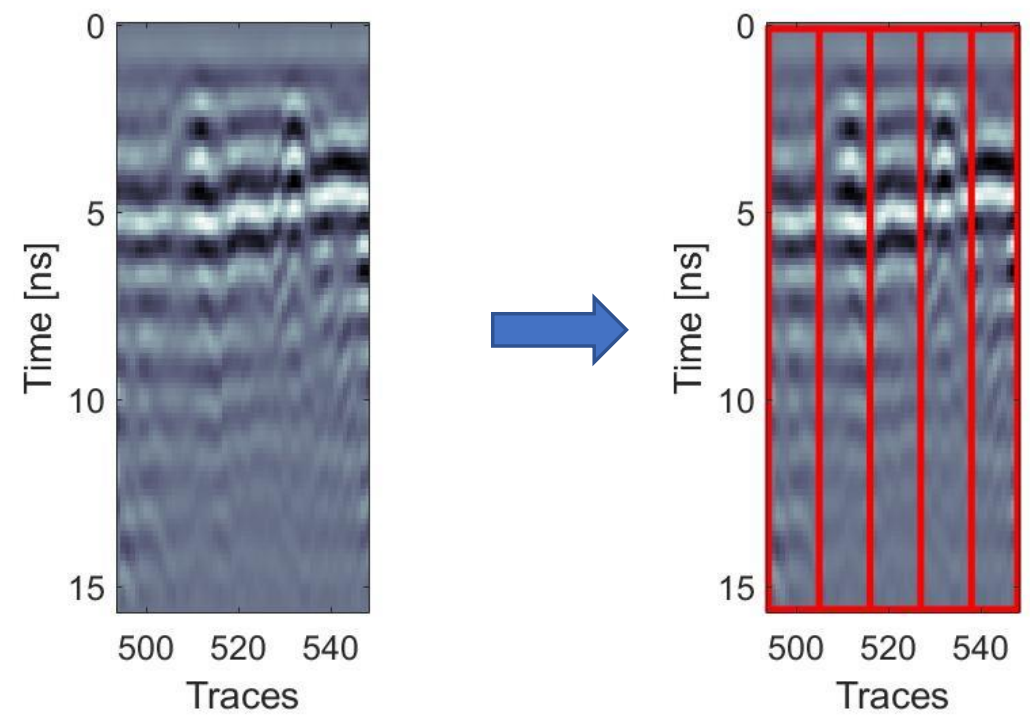

Figure 3. The selected area (left) and its partition into application windows. 


\subsection{Application of the STFT Function}

Following the pre-processing phase, the STFT function was applied to the application windows' average trace. Figure 4 shows the resulting time-frequency analysis carried out using this approach.

1

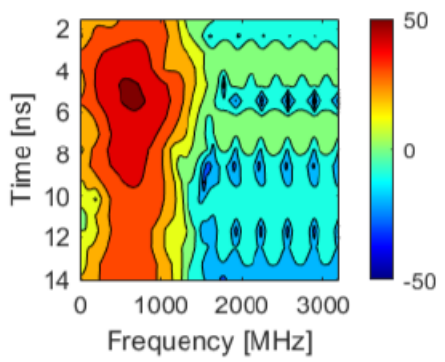

2



3

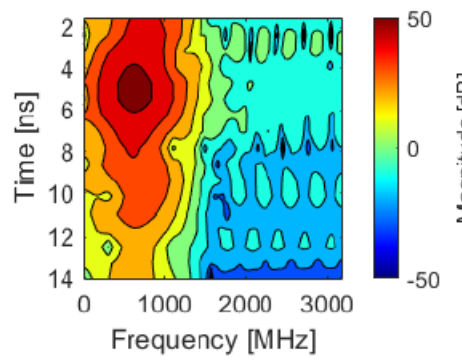

4

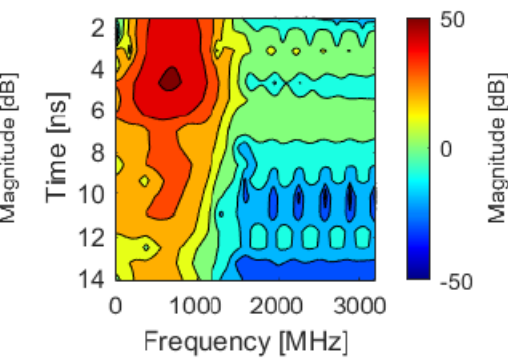

5



Figure 4. STFT outputs for the selected application windows

From the analysis of the STFT outputs of windows 2 and 4 (which are related to the reflection hyperbolas), it is evident how the energy peaks are concentrated in peaks centred at an arrival time of about $5 \mathrm{~ns}$ for window 2 and 4 ns for window 4. The centroids of such energy peaks, located in a frequency range between 500 and $700 \mathrm{MHz}$, are in both cases of small dimensions and regular shape. Also, in both cases it can be noted that the energy rapidly decreases between 11 and $12 \mathrm{~ns}$, with a sudden drop in case of window 4, likely related to a hyperbola with smaller dimension and greater amplitude than the one in window 2. This proves that most of the energy is concentrated in the position of the hyperbolas, and that the STFT method is capable to identify the presence of tree roots correctly even in the presence of a horizontal layer that intersects them.

As for the STFT outputs 1 and 5, i.e., those referring to the layer's reflections only, the energy peaks are concentrated at an arrival time of about $5 \mathrm{~ns}$ and $4 \mathrm{~ns}$, respectively. It is worth noting how the energy peaks have an irregular shape and can have a significant size, as in the case of window 5 where the peak is located in a frequency range between 300 and 900 $\mathrm{MHz}$. Also, in both cases the energy decreases less rapidly, so much that at an arrival time of 14 ns the magnitude remains stable at around $40 \mathrm{~dB}$.

Finally, regarding the STFT output 3, which refers to the window between the two investigated hyperbolas, the attenuation of the energy is not substantially different from the ones of windows 2 and 4. Also, the shape of the energy peak centroid is fairly regular. Such response could be misleading, as they present similar features to those observed in the case of spectra with presence of hyperbolas. This could be due to the fact that window 3 is located between two hyperbolas in close proximity, and the reflection of the pavement layer could have been magnified by the superimposition of these hyperbolas' tails. Nevertheless, it is worth noting how the energy peak centroid covers a wider frequency range than the windows containing the hyperbolas' apices. Furthermore, while for higher frequencies (i.e. $1500 \mathrm{MHz}$ and above) the hyperbolarelated spectra show a similar pattern and a positive magnitude, the spectrum of window 3 has a different shape and a negative magnitude of around $-10 \mathrm{~dB}$. 


\subsection{Error Maps}

To further investigate the differences between different STFT outputs, error maps have been produced. These were given by the difference between the outputs of the examined map and those of a reference map. As a representative scenario, Figure 5 shows the error maps created for the investigated application windows, with reference to the window 2 STFT output.
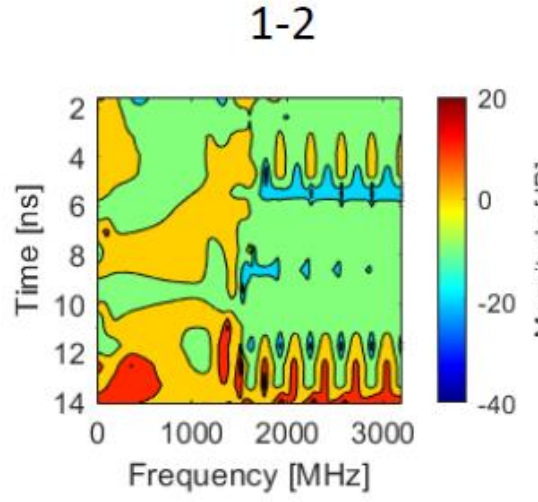

4-2



3-2

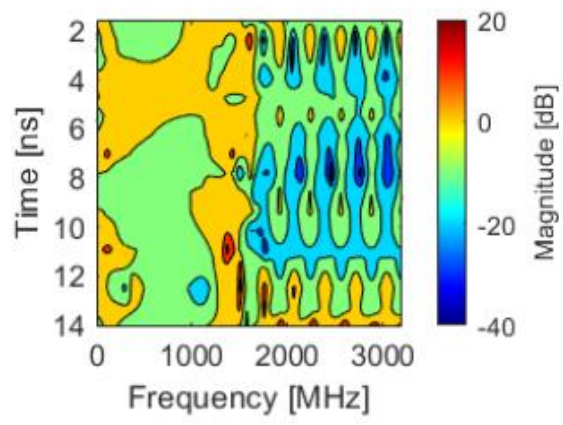

$5-2$

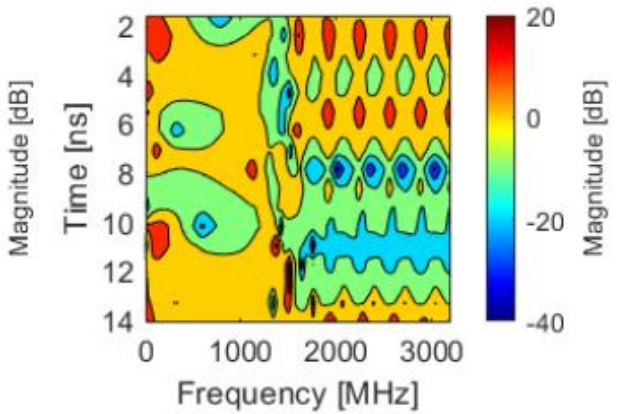

Figure 5. Error maps for the STFT spectra, with reference to the window 2 STFT spectra.

It is worth noting how the 4-2 error map, i.e., the difference between the hyperbola in window 4 and the hyperbola in window 2, shows a different pattern compared to the rest of scenarios. In fact, an area of negative magnitude extends uninterrupted from an arrival time of $2 \mathrm{~ns}$ up to $14 \mathrm{~ns}$, with a negative peak ranging from about 7 to 9 ns. Conversely, in all the other error maps it is possible to see an alternation of areas with positive and negative magnitude, covering the range of frequencies from 0 to about $1000 \mathrm{MHz}$. In particular, when comparing window 2 with the others, it is noteworthy that an area of positive error is always present for lower frequencies and arrival times greater than 10ns (and also for early arrival times in error map 5-2). This is particularly evident for maps 1-2 and 5-2 (i.e., difference between the hyperbola and the layer), while is very limited for windows 3-2 and 4-2.

To further investigate this trend, and to observe the trend of the error maps' characteristics compared with the response of the time-frequency analysis in the presence of a root, the spectra of the energy in the error maps were evaluated. A time window was therefore selected, with size comparable to the reference hyperbola (i.e., between 2 and 4.5 ns), as shown in Figure 6. The average spectra trend of the error maps within the selected time window is shown in Figure 7. 


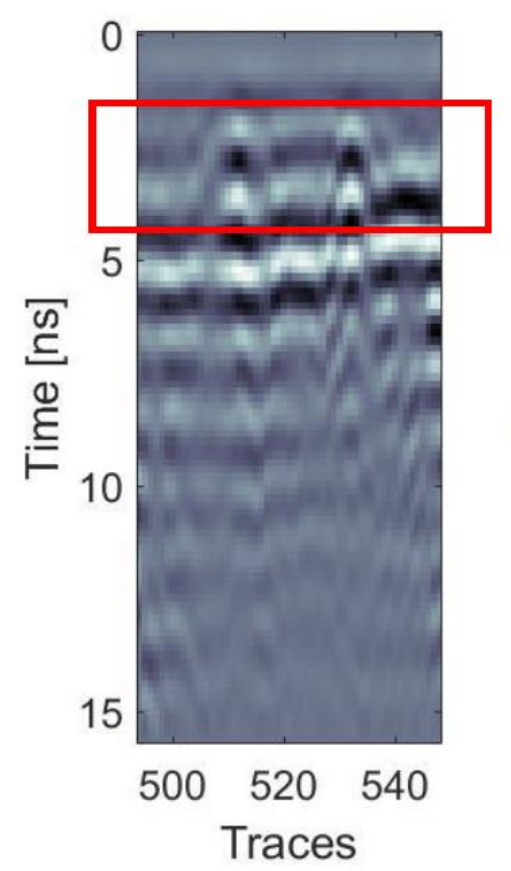

$1-2$

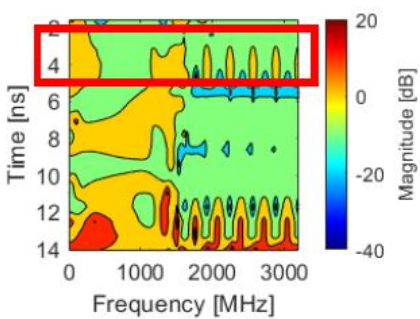

4-2



$3-2$

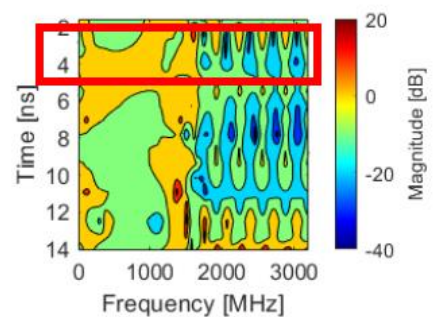

$5-2$

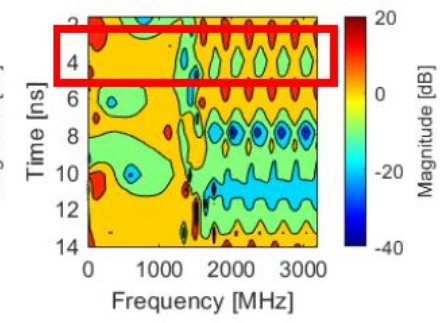

Figure 6. Identification of the time window for the analysis of the error maps' spectra trend

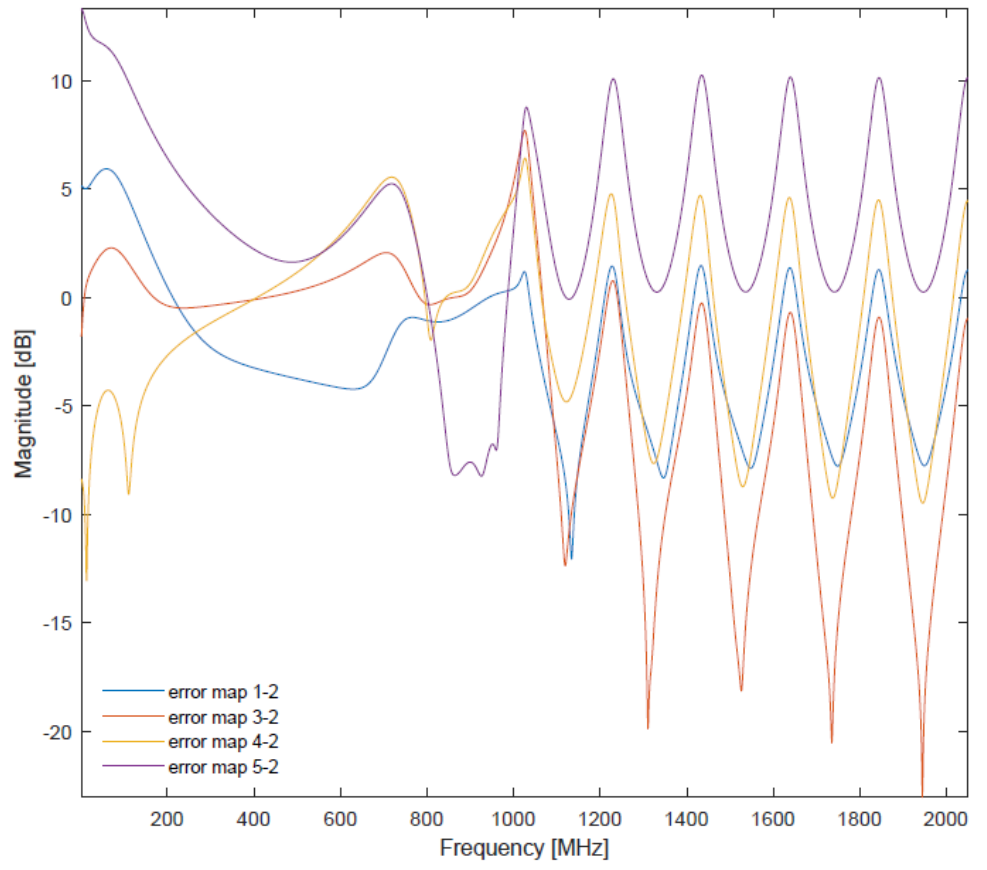

Figure 7. Average spectra trend of error maps between 2 and $4.5 \mathrm{~ns}$

It is evident that all the error maps' average spectra have a similar trend, decreasing towards $600 \mathrm{MHz}$ and rising again to form a peak around $700 \mathrm{MHz}$. The only spectrum not following this trend is the one related to the difference between the two hyperbolas. In fact, in this case the trend rises up from a negative magnitude, forming an ascending trend and then a peak at $700 \mathrm{MHz}$. This demonstrates that the methodology is capable of recognising hyperbolic features related to tree roots, and to distinguish them from the pavement layers in which these are embedded. 


\section{CONCLUSIONS AND FUTURE PERSPECTIVES}

This study shows the latest developments of a novel approach for detection of tree roots in urban environments with Ground Penetrating Radar (GPR) technology. To this end, a Short-Time Fourier Transform (STFT), based on the analysis of data in both time and frequency domain combined, was applied to GPR data collected on urban trees, following a pre-processing stage aimed at removing both noise-related information and disturbances caused by manmade features, such as pavement layers.

Results demonstrated the effectiveness of the presented approach in identifying signal features associated to tree roots and clearly distinguish them from the response obtained by the surrounding pavement layers. The analyses of the data have showed recurring patterns in the STFT outputs, which can be connected to different features (i.e., roots, pavement, etc.). The outcomes of this research confirm that the presented method can be suitable for use in urban environments, allowing to assess and monitor street trees in a rapid and non-invasive way. Further investigations and developments are envisaged to minimise risk of false alarms and automate the procedure, thus paving the way for a more systematic monitoring process with scopes in terms of protection of urban trees, the maintenance of the built environment and the safety of its users.

\section{REFERENCES}

[1] United Nations, Department of Economic and Social Affairs, Population Division , "World Urbanization Prospects: The 2014 Revision," (2015).

[2] Donovan, G. H., Butry, D. T., Michael, Y. L., Prestemon, J. P., Liebhold, A. M., Gatziolis, D. and Mao, M. Y. , "The relationship between trees and human health: evidence from the spread of the emerald ash borer," American journal of preventive medicine 44(2), 139-145 (2013).

[3] van Dillen, Sonja M E, de Vries, S., Groenewegen, P. P. and Spreeuwenberg, P. , "Greenspace in urban neighbourhoods and residents' health: adding quality to quantity," Journal of Epidemiology and Community Health 66(6) (2012).

[4] Mullaney, J., Lucke, T. and Trueman, S. J., "A review of benefits and challenges in growing street trees in paved urban environments," Landscape and Urban Planning 134, 157-166 (2015).

[5] BBC, "More than 50,000 urban trees to be planted in England," (2020).

[6] Day, R. W. , "Damage of Structures due to Tree Roots," Journal of performance of constructed facilities 5(3), 200207 (1991).

[7] Brennan, G., Patch, D. and Stevens, F. R. W. , "Tree Roots and Underground Pipes - Arboriculture Research Note," (1997).

[8] Mullaney, J., Lucke, T. and Trueman, S. J., "A review of benefits and challenges in growing street trees in paved urban environments," Landscape and urban planning 134, 157-166 (2015).

[9] Alani, A. M., Aboutalebi, M. and Kilic, G. , "Applications of ground penetrating radar (GPR) in bridge deck monitoring and assessment," Journal of applied geophysics 97, 45-54 (2013).

[10] Gonzalez-Huici, M. A., Catapano, I. and Soldovieri, F. , "A Comparative Study of GPR Reconstruction Approaches for Landmine Detection," JSTARS 7(12), 4869-4878 (2014). 
[11] Tosti, F., Adabi, S., Pajewski, L., Schettini, G. and Benedetto, A., "Large-scale analysis of dielectric and mechanical properties of pavement using GPR and LFWD," Proceedings of the 15th International Conference on Ground Penetrating Radar, 868-873 (Jun 2014).

[12] Benedetto, A., Tosti, F., Ciampoli, L. B., Pajewski, L., Pirrone, D., Umiliaco, A. and Brancadoro, M. G., "A simulation-based approach for railway applications using GPR," 2016 16th International Conference on Ground Penetrating Radar (GPR), 1-6 (Jun 2016).

[13] Hruska, J., Čermák, J. and Šustek, S. , "Mapping tree root systems with ground-penetrating radar," Tree Physiol. 19(2), 125-130 (1999).

[14] Barton, C. V. M. and Montagu, K. D. , "Detection of tree roots and determination of root diameters by ground penetrating radar under optimal conditions," Tree physiology 24(12), 1323-1331 (2004).

[15] Butnor, J. R., Doolittle, J. A., Johnsen, K. H., Samuelson, L., Stokes, T. and Kress, L. , "Utility of groundpenetrating radar as a root biomass survey tool in forest systems," Soil Sci.Soc.Am.J. 67(5), 1607-1615 (2003).

[16] Alani, A. M., Ciampoli, L. B., Lantini, L., Tosti, F. and Benedetto, A., "Mapping the root system of matured trees using ground penetrating radar," 2018 17th International Conference on Ground Penetrating Radar (GPR), 1-6 (2018).

[17] Lantini, L., Tosti, F., Giannakis, I., Zou, L., Benedetto, A. and Alani, A. M. , "An Enhanced Data Processing Framework for Mapping Tree Root Systems Using Ground Penetrating Radar," Remote Sensing 12(20), 3417 (2020).

[18] Lantini, L., Tosti, F., Giannakis, I., Zou, L., Egyir, D., Mortimer, D. and Alani, A. M., "Health assessment of trees using GPR-derived root density maps," 2020 IEEE Radar Conference (RadarConf20), 1-5 (Sep 21, 2020).

[19] Lantini, L., Alani, A., Giannakis, I., Benedetto, A. and Tosti, F. , "Application of ground penetrating radar for mapping tree root system architecture and mass density of street trees," Advances in Transportation Studies(3), 51-62 (2019).

[20] Lantini, L., Tosti, F., Bianchini Ciampoli, L. and Alani, A. M., "A frequency spectrum-based processing framework for the assessment of tree root systems,", 115251J-7 (Nov 8, 2020).

[21] Randrup, T. B., McPherson, E. G. and Costello, L. R. , "A review of tree root conflicts with sidewalks, curbs, and roads," Urban Ecosystems 5(3), 209-225 (2001).

[22] Tosti, F., Bianchini Ciampoli, L., Brancadoro, M. G. and Alani, A. , "GPR applications in mapping the subsurface root system of street trees with road safety-critical implications," Advances in Transportation Studies (2018). 